NOUVELLE

\section{Le microbiote : promoteur de la différenciation des lymphocytes T CD8 mémoires}

Anthony Mainguy ${ }^{1}$, Clara-Eva Paquereau ${ }^{2}$, Paul Stys $^{1}$, Signe Hässler ${ }^{3}$
> Le microbiote désigne l'ensemble des micro-organismes tels que les bactéries, les champignons, les virus et les parasites non pathogènes qui colonisent les surfaces de l'hôte en contact avec le milieu extérieur. L'émergence des études du microbiote a permis de mettre en avant son influence sur le fonctionnement des défenses immunitaires de l'hôte. Le système immunitaire $(\mathrm{SI})$ est divisé en deux parties: l'immunité innée, immédiate mais peu spécifique, ainsi que l'immunité adaptative, plus lente à se mettre en place mais plus efficace par son mécanisme d'action, le ciblage antigénique.

Les lymphocytes T (LT) forment une population hétérogène de cellules immunitaires impliquées dans les réponses adaptatives. Les précurseurs hématopoïétiques sont issus de la moelle osseuse, ils poursuivent leur développement dans le thymus puis résident à l'état naïf dans les organes lymphoïdes secondaires, en attente d'une activation antigénique.

Les LT se subdivisent en deux grands groupes cellulaires: les LT CD4 ${ }^{+}$auxiliaires qui participent à l'activation de certaines cellules de l'immunité (lymphocytes $B, L T C D 8^{+}$, macrophages, etc.) ainsi qu'à l'enclenchement de l'immunité à médiation humorale, tandis que les $\mathrm{LT} \mathrm{CD}^{+}$sont impliqués dans la réponse à médiation cellulaire grâce à leur activité cytotoxique. De nombreuses souspopulations existent au sein de ces deux groupes, comme les $\mathrm{LT} \mathrm{CD}^{+}$régulateurs ( $\left.T_{R E G}\right)$ issus du développement thymique ou d'une différenciation plus tardive en périphérie, ainsi que les LT mémoires $\left(T_{M}\right)$ issus des cellules effectrices. Les $T_{R \varepsilon G}$ ont un rôle immunosuppresseur et peuvent être assimilés à des modulateurs du système immunitaire. Les $T_{M}$ sont des cellules à longue durée de vie, indispensables à l'activation du SI en cas de seconde infection par le même agent pathogène.

\section{Relation entre immunité et microbiote}

La comparaison entre des modèles murins dépourvus de la totalité de leur microbiote (GF pour germ-free), ou d'un seul ou plusieurs pathogènes spécifiques (SPF pour specific pathogen-free) a permis d'étudier les interactions entre le microbiote et l'immunité de l'hôte. Une absence de flore intestinale est associée à un déficit immunitaire, caractérisé par un défaut de différenciation et de prolifération des $T_{R \varepsilon G} C D^{+}[1,2]$. De plus, le microbiote est impliqué dans l'activation et l'activité cytotoxique des $\mathrm{LT} \mathrm{CD8} 8^{+}$[3] ainsi que dans la différenciation et le métabolisme des $T_{M} C D 8^{+}$ [4]. Afin d'expliquer cet impact sur le SI, certaines études se sont intéressées en particulier aux fonctions métaboliques assurées par le microbiote. Ainsi, il a été démontré que la fermentation de fibres alimentaires impliquait l'intervention de souches bactériennes particulières et aboutissait à la formation de certains métabolites, dont les acides gras à chaîne courte (AGCC) [1].

Une étude récente s'est particulièrement intéressée au potentiel rôle des AGCC dans la différenciation des LT CD8 ${ }^{+}$ en cellules mémoires [5]. Dans cette étude, des LT spécifiques d'une glycoprotéine du virus Herpès simplex (HSV) et activés préalablement in vitro avec l'antigène correspondant, sont injec-
${ }^{1}$ Master 2 Immunologie Systémique et Intégrative (i2s), Mention BMC, Sorbonne Université, Paris, France.

${ }^{2}$ Master 2 Immunologie Translationnelle et Biothérapie (ITB), Mention BMC, Sorbonne Université, Paris, France.

${ }^{3}$ Sorbonne Université, UFR927 et Sorbonne Université, Inserm UMR 959, ImmunologieImmunopathologie-Immunotherapie (i3), Paris, France.

anthony-mainguy@hotmail.fr claraeva.paquereau@gmail.com paul.stys@wanadoo.fr signe.hassler@sorbonne-universite.fr

tés par voie sanguine dans des souris SPF soumises ou non à un régime riche en fibres pendant les cinq semaines précédant l'injection des LT. La poursuite d'un régime riche en fibres intensifie la réponse mémoire développée après une restimulation avec l'antigène HSV. Chez les souris suivant ce régime particulier, cette réponse mémoire est caractérisée par une meilleure prolifération des $\mathrm{LT} \mathrm{CD8}^{+}$effecteurs dérivant des $\mathrm{T}_{M} C D 8^{+}$, ainsi que par une production accrue de médiateurs solubles tels que I'IFN(interféron)- $\gamma$, une cytokine impliquée dans la réponse antivirale [5]. De plus, d'autres expériences in vivo effectuées dans le cadre de cette étude montrent que la réponse mémoire de LT CD $8^{+}$déficients pour les récepteurs GPR ( $G$ protein-coupled receptors) 41 et GPR43, spécifiques des AGCC, est plus faible que celle de $\mathrm{LT} C D 8^{+}$exprimant ces récepteurs. Ces expériences suggèrent ainsi que les effets cellulaires des AGCC nécessitent une interaction avec ces récepteurs [5].

Le butyrate favorise la différenciation des LT CD8 ${ }^{+}$en cellules mémoires

Le suivi d'un régime riche en fibres a pour conséquence d'augmenter d'un facteur 100 la concentration sanguine du butyrate, un AGCC. Pour mimer les conditions observées in vivo, des $\mathrm{LT} C \mathrm{CD} 8^{+}$ont été exposés in vitro à un taux élevé de butyrate. Les cellules ainsi stimulées montrent une meilleure capacité de réponse à I'IL(interleukine)-15 et sur-expriment le facteur de transcription Fox01. Ce fac- 


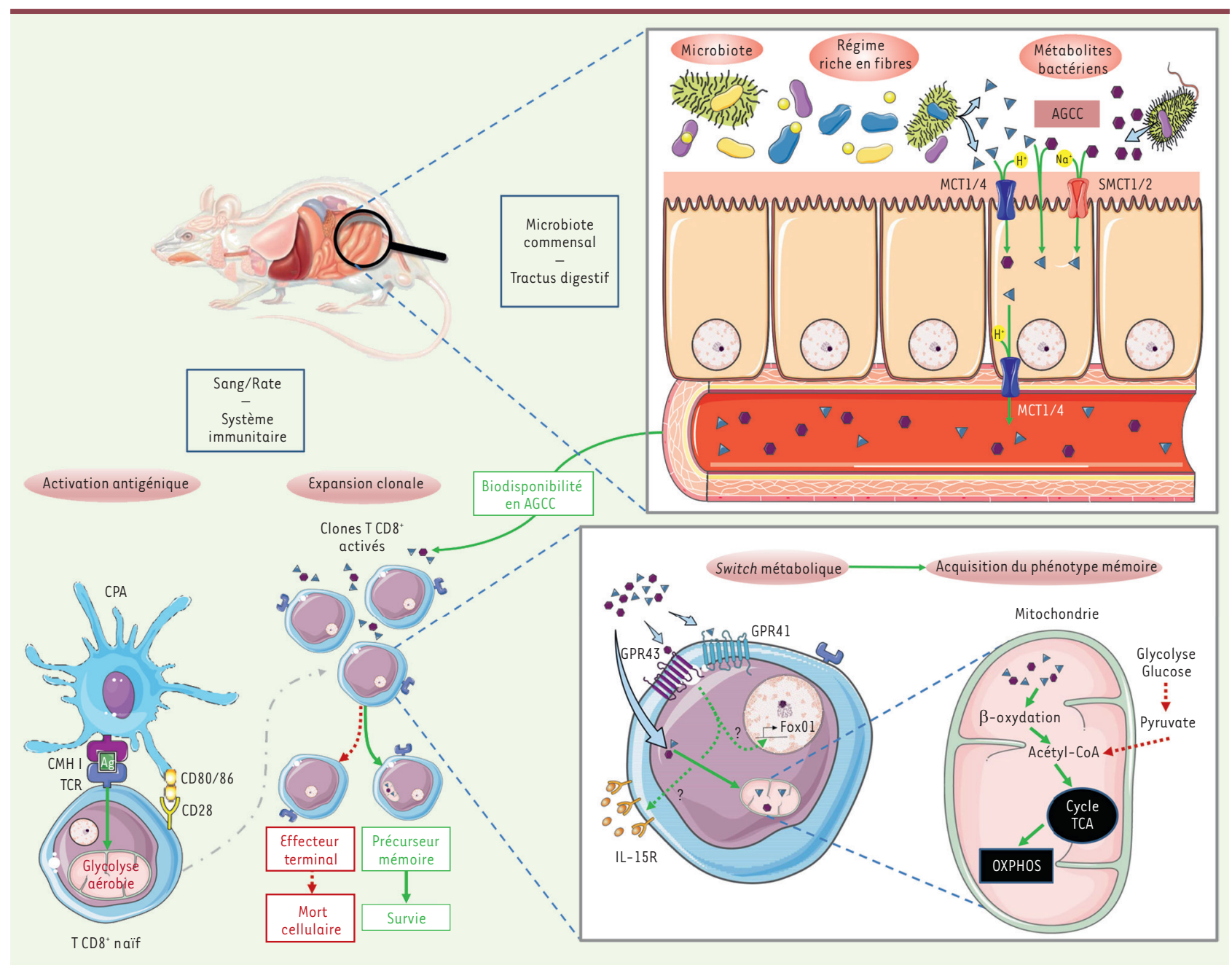

Figure 1. Implication des acides gras à chaînes courtes issus du métabolisme des bactéries microbiotiques dans l'induction d'un phénotype mémoire des lymphocytes $T C D 8^{+}$. Les AGCC peuvent passer l'épithélium intestinal et se retrouver dans la circulation sanguine où ils peuvent être utilisés par les cellules. Cette absorption s'effectue de manière passive au travers de la membrane épithéliale, ou active par le biais des co-transporteurs ioniques. Les AGCC assimilés par les LT CD8 ${ }^{+}$induisent des modifications métaboliques privilégiant la $\beta$-oxydation des acides gras. Ce switch métabolique participe à la différenciation en LT mémoire. AGCC : acide gras à chaînes courtes ; MCTl et MCT4 : monocarboxylate transporter 1 et 4 ; SMCT1 et SMCT2 : sodium-coupled monocarboxylate transporter 1 et 2 ; CPA : cellule présentatrice d'antigène ; GPR43 et GPR41 : récepteur aux AGCC $43 / 41$; cycle TCA : cycle de Krebs; OXPHOS : phosphorylation oxydative.

teur est impliqué dans la modulation du métabolisme énergétique cellulaire et est caractéristique de la différenciation en cellules mémoires [4]. Simultanément, la prolifération des $\mathrm{T} C D 8^{+}$activés est réduite. Ces différentes caractéristiques correspondent à la description d'un phénotype de cellules mémoires [5].

Des analyses transcriptomiques comparant les profils d'expression génique des $L T C D 8^{+}$spécifiques activés transférés dans des souris SPF ou des LT CD $8^{+}$ transférés dans des souris GF ont mon- tré qu'une centaine de gènes impliqués dans la différenciation mémoire sont significativement surexprimés dans les $\mathrm{T} C D 8^{+}$transférés dans les souris SPF. $\varepsilon$ n lien avec ce résultat, le transcriptome des $\mathrm{LT} \mathrm{CD}^{+}$cultivés dans un milieu enrichi en butyrate montre également une augmentation de l'expression de ces gènes. Une partie des gènes surexprimés dans les $\mathrm{LT} C D 8^{+}$transférés dans les souris SPF codent notamment des protéines de complexes mitochondriaux et des transporteurs d'électrons, ce qui suggère un lien entre le microbiote (et par extension la présence d'AGCC) et la différenciation des $\mathrm{LT} \mathrm{CD}^{+}$en cellules mémoires via une modulation du métabolisme énergétique de ces cellules [5].

\section{Profil T et signature métabolique}

La production d'adénosine triphosphate (ATP) est une fonction essentielle de toutes les cellules de notre organisme, y compris des LT. Cette production est issue de différentes voies métaboliques en fonction de la biodisponibilité des 
substrats énergétiques dans le microenvironnement cellulaire. De nombreuses études ont démontré l'existence de changements dynamiques dans le métabolisme énergétique des lymphocytes $T$ selon leur état d'activation et leur stade de différenciation. Ainsi, une cellule activée à la suite d'une rencontre antigénique va privilégier un métabolisme centré sur l'utilisation du glucose et l'accumulation d'une biomasse nécessaire à la prolifération cellulaire (glycolyse aérobie), tandis qu'une cellule naïve quiescente sera caractérisée par un métabolisme basal globalement nonglycolytique [6].

Après résolution d'une infection, le système immunitaire subit une phase de contraction correspondant à une réduction significative du nombre de lymphoytes $T$ effecteurs $\left(T_{\varepsilon F F}\right)$. II s'ensuit une transition vers le phénotype $T_{M}$, gouvernée par des mécanismes complexes partiellement élucidés à l'heure actuelle. Cependant, des études ont apporté quelques précisions quant au rôle du métabolisme énergétique dans l'induction des $T_{M}$. En effet, une communication (switch), métabolique, passant d'une production quasi-exclusivement glucose-dépendante (biosynthèse) à un métabolisme fondé sur l'oxydation d'acide gras (AG) ( $\beta$-oxydation ou FAO, fat acid oxydation) faciliterait cette transition. La FAO permet la production d'un substrat énergétique directement incorporable dans le cycle de Krebs: l'acétyl-CoA. Ce substrat est indispensable pour générer les transporteurs d'électrons, le FADH2 et le NADH utilisés dans la chaîne respiratoire mitochondriale lors de la phosphorylation oxydative, réaction aboutissant à la bio-synthèse d'ATP [7].

Comme nous l'avons présenté auparavant, la $\beta$-oxydation des $A G$ est une voie privilégiée dans la transition des $T_{\varepsilon F F}$ en $T_{M}$. De ce fait, le microbiote pourrait fournir une source de substrat à base d'AGCC, facilitant cette transition $T_{\varepsilon F F}$ en $T_{M}$. Pour tester cette hypothèse, des $\mathrm{LT} C \mathrm{CD} 8^{+}$activés ont été mis en culture avec du butyrate marqué au ${ }^{13} \mathrm{C}$ afin de suivre son incorporation dans les différentes voies métaboliques cellulaires. Ce dernier est retrouvé en quantité importante dans tous les métabolites intermédiaires du cycle de Krebs [5]. Cela confirme donc que le butyrate, et par extension les AGCC, peuvent servir de substrat énergétique aux $\mathrm{LT} C D 8^{+}$et induire de façon directe ou indirecte un switch métabolique participant à la différenciation des $T_{\varepsilon F F}$ en $T_{M}$ comme décrit précédemment.

Le microbiote est un système complexe assurant l'immuno-modulation des réponses immunitaires des LT. En effet, ces récents résultats démontrent son implication dans la différenciation et la prolifération des lymphocytes T CD8 ${ }^{+}$ mémoires via les AGCC issus de la fermentation microbiotique de fibres. Cette propriété des médiateurs solubles offre des pistes intéressantes pour l'optimisation des stratégies vaccinales. Alors que la recherche actuelle se focalise principalement sur l'évolution des vecteurs vaccinaux ou l'usage d'adjuvants, les produits du microbiote pourraient jouer un rôle dans l'établissement d'une mémoire $\mathrm{T} C D 8^{+}$protectrice durable suite à une vaccination [5]. $\diamond$

Microbiota: inducer of memory CD8 ${ }^{*} \mathrm{~T}$ lymphocyte differentiation?

\section{LIENS D'INTÉRÊT}

Les auteurs déclarent n'avoir aucun lien d'intérêt concernant les données publiées dans cet article.

\section{RÉFÉRENCES}

1. Arpaia N, Campbell C, Fan X, et al. Metabolites produced by commensal bacteria promote peripheral regulatory T-cell generation. Nature 2013 ; 504 : 451-5.

2. Smith PM, Howitt MR, Panikov N, et al. The microbial metabolites, short-chain fatty acids, regulate colonic Treg cell homeostasis. Science. 2013 ; 341 : 569-73.

3. Trompette A, Gollwitzer ES, Pattaroni C, et al. Dietary fiber confers protection against flu by shaping Ly6cpatrolling monocyte hematopoiesis and $\mathrm{CD} 8^{+} \mathrm{T}$ cell metabolism. Immunity 2018; 48 : 992-1005.

4. Kazuo T, Sadaaki S, Satoh T, et al. Role of the indigenous microbiota in maintaining the virusspecific CD8 memory T cells in the lung of mice infected with murine cytomegalovirus. J Immunol $2007 ; 178: 5209-16$.

5. Bachem A, Makhlouf C, Binger KJ, et al. Microbiotaderived short-chain fatty acids promote the memory potential of antigen-activated $\mathrm{CD} 8^{+} \mathrm{T}$ cells. Immunity $2019 ; 51: 285-97$

6. Greiner EF, Guppy M, Brand K. Glucose is essential for proliferation and the glycolytic enzyme induction that provokes a transition to glycolytic energy production. J Biol Chem 1994 ; 269 : 31484-90.

7. Pearce EL, Walsh MC, Cejas PJ, et al. Enhancing CD8 $T$-cell memory by modulating fatty acid metabolism. Nature 2009 ; 460 : 103-7.

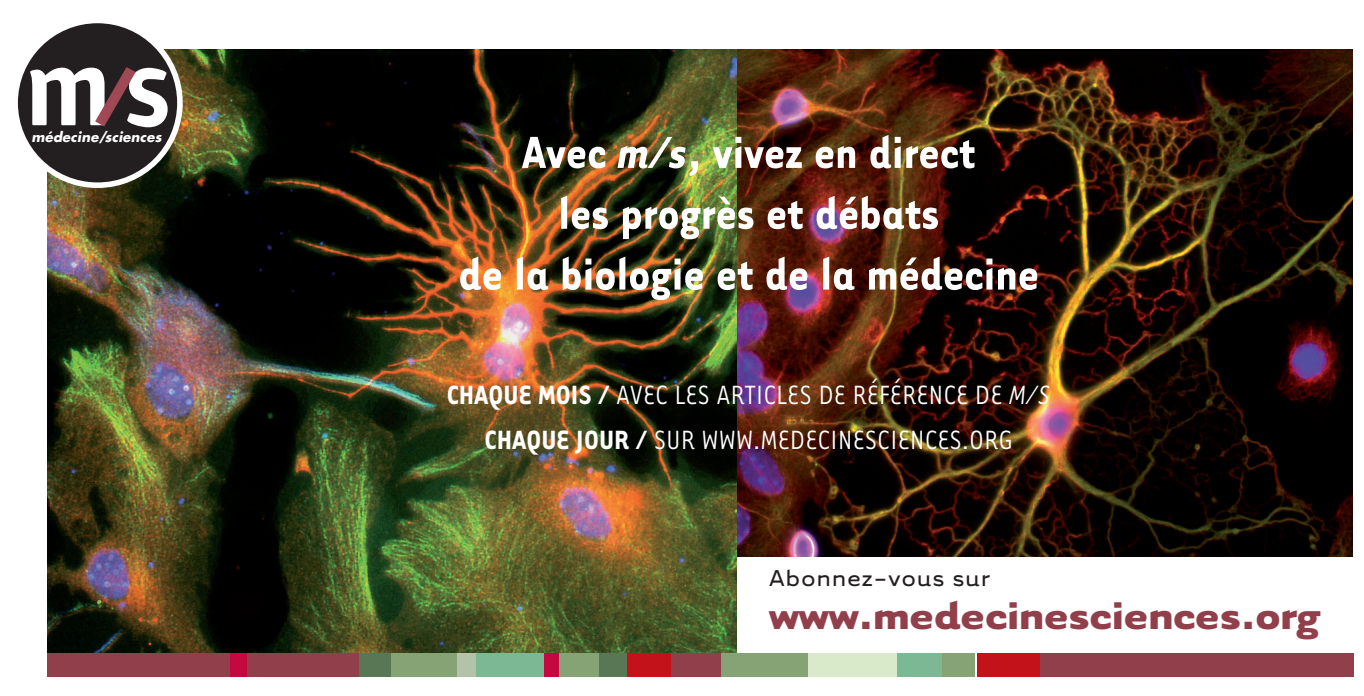

